
August 2003
Volume 112
Number 8

COPYRIGHTC 2003, ANNALS PUBLISHING COMPANY

\title{
TREATMENT OF INFERIOR TURBINATE HYPERTROPHY: A RANDOMIZED CLINICAL TRIAL
}

\author{
DESIDERIO PASSÀLI, MD, PHD \\ SIENA, ITALY \\ FRANCESCO MARIA PASSÀLI, MD \\ GENOA, ITALY \\ GiUlio CeSARE PASSÀli, MD \\ SIENA, ITALY \\ VALERIO DAMIANI, MD \\ SIENA, ITALY \\ LUISA BELLUSSI, MD, CHD \\ SIENA, ITALY
}

In the past 130 years, many surgical procedures for turbinate reduction have been developed. We analyzed the long-term efficacy of 6 of these surgical techniques (turbinectomy, laser cautery, electrocautery, cryotherapy, submucosal resection, and submucosal resection with lateral displacement) over a 6-year follow-up period. We randomly divided 382 patients into 6 therapeutic groups and surgically treated them at the Department of Otorhinolaryngology of the University of Siena. After 6 years, only submucosal resection resulted in optimal long-term normalization of nasal patency and in restoration of mucociliary clearance and local secretory IgA production to a physiological level with few postoperative complications $(\mathrm{p}<.001)$. The addition of lateral displacement of the inferior turbinate improved the long-term results. We recommend, in spite of the greater surgical skill required, submucosal resection combined with lateral displacement as the first-choice technique for the treatment of nasal obstruction due to hypertrophy of the inferior turbinates.

KEY WORDS - hypertrophy, inferior turbinate, submucosal resection.

\section{INTRODUCTION}

The role of inferior turbinate hypertrophy in the reduction of nasal airflow is well established, representing, if septal deviations are excluded, the main cause of symptomatic nasal obstruction. ${ }^{1}$ Although chronic nasal obstruction is not life-threatening, it impairs patients' quality of life in a significant way, affecting many aspects of their daily social and working activities. ${ }^{2}$

Perennial allergic rhinitis and nonallergic rhinitis are the most common noninfectious causes of inferior turbinate mucosal swelling, leading to a transient reduction of nasal patency. Many of these cases respond to medical treatment with topical corticosteroids, antihistamines, or immunotherapy. In some patients, however, these nasal inflammatory processes result in chronic nasal obstruction due to dilatation of the venous sinuses or fibrosis, ${ }^{3}$ and as a consequence, medical therapy is not sufficient. In these cases, a surgical approach to the enlarged inferior, and sometimes middle, turbinates becomes necessary.

Since the first surgical procedure for turbinate reduction, performed by Hartmann in the 1890s, many other techniques have been developed. ${ }^{4}$ Of these, the most widely used are turbinectomy, ${ }^{5,6}$ laser cautery, 7,8 electrocautery, 9,10 cryotherapy, ${ }^{11,12}$ submucosal resection, ${ }^{13,14}$ and submucosal resection with lateral displacement. ${ }^{15,16}$ None of these procedures is ideal; each is associated with known short- and long-term complications. The lack of consensus about their effectiveness leaves selection of the surgical procedure up to the surgeon's personal attitude and experience. To provide evidence to critically assess the outcomes of the different surgical techniques, we compared the long-term efficacy of 6 surgical techniques for turbinate reduction over a 6-year follow-up period.

\section{MATERIALS AND METHODS}

We identified 457 patients (256 male and $201 \mathrm{fe}-$ male) between 8 and 70 years of age (median age, 38 years) with symptoms of nasal obstruction. Each patient underwent a diagnostic protocol consisting of clinical history-taking, otorhinolaryngological objective examination, skin prick tests for allergy (grass pollen, Parietaria, and Dermatophagoides), anterior active rhinomanometry with the Amplifon Rhynosystem, acoustic rhinometry with a Rhinoklack RK 1000, analysis of nasal mucociliary transport time by measurement of the time required for charcoal powder placed on the inferior turbinate to reach the pharynx, ${ }^{17}$ and measurement of secretory immunoglobulins.

From the Department of Otorhinolaryngology, University of Siena, Medical School, Siena (D. Passàli, Damiani, G. C. Passàli, Bellussi), and the Department of Otorhinolaryngology, University of Genoa, Medical School, Genoa (F. M. Passàli), Italy.

CORRESPONDENCE - Desiderio Passàli, MD, PhD, Dept of Otorhinolaryngology, University of Siena, Medical School, V.le Bracci, 53100 Siena, Italy. 
TABLE 1. POPULATION DATA

\begin{tabular}{lccc}
\hline \hline Treatment & $\begin{array}{c}\text { No. of } \\
\text { Patients }\end{array}$ & Male & Female \\
\hline Turbinectomy & 45 & 24 & 21 \\
Laser cautery & 54 & 28 & 26 \\
Electrocautery & 62 & 32 & 30 \\
Cryotherapy & 58 & 30 & 28 \\
Submucosal resection & 69 & 37 & 32 \\
Submucosal resection with & 94 & 50 & 44 \\
$\quad$ lateral displacement & & & \\
All treatments & 382 & 201 & 181 \\
\hline
\end{tabular}

The patients graded their symptoms of nasal obstruction during the day and night, nasal discharge during the day and night, and sensation of ear fullness on a scale of 1 to 6 using validated questionnaires; a total symptom score was obtained by adding the 5 values.

Of the 457 patients evaluated, 382 were enrolled in the surgical phase of the study (201 male and 181 female) on the basis of the inclusion criteria of the presence of chronic allergic or vasomotor rhinitis leading to chronic nasal obstruction and a lack of efficacy of medical treatment.

The exclusion criteria were presence of infectious rhinitis, marked septal deviation, previous nasal surgery, nasal polyps or sinusitis contributing to the nasal obstruction, and other major nasal diseases.

All of the enrolled patients were treated at the Department of Otorhinolaryngology of the University of Siena. The following surgical procedures were performed: turbinectomy, laser cautery, electrocautery, cryotherapy, submucosal resection, and submucosal resection with lateral displacement. All of the surgical procedures were carried out under local anesthesia.

Turbinectomy. After medial and upward fracture, the inferior turbinate was resected by angled scissors along the insertion close to the lateral nasal wall. Merocel nasal packing was used for 3 days.

Laser Cautery. The inferior turbinates were coagulated with a $\mathrm{CO}_{2}$ laser (Coherent MP20 laser) delivering 300 impulses per second at 10 to $15 \mathrm{~W}$. To
TABLE 2. COMPLICATIONS

\begin{tabular}{lcccc}
\hline \hline Treatment & Crusting & Synechiae & Bleeding & Atrophy \\
\hline Turbinectomy & 34 & 14 & 25 & 10 \\
Laser cautery & 40 & 4 & 0 & 6 \\
Electrocautery & 39 & 21 & 0 & 2 \\
Cryotherapy & 40 & 8 & 0 & 3 \\
Submucosal & 7 & 2 & 10 & 0 \\
$\quad$ resection & & & & \\
Submucosal & 6 & 2 & 8 & 0 \\
$\quad$ resection & & & & \\
$\quad$ with lateral & & & & \\
$\quad$ displacement & & & & \\
$\quad$ Data are numbers of patients. & &
\end{tabular}

avoid excessive carbonization, we left a $2.5-\mathrm{ms}$ interval between consecutive applications. Packing was not necessary.

Electrocautery. Inferior turbinate cauterization was done by applying a high-frequency coagulation current via a round-point electrode at constant power introduced as far as the posterior end of the nasal cavity. The medial surface of the inferior turbinate was coagulated from behind forward, 2 to 4 times, $10 \mathrm{sec}-$ onds each time. At the end of the intervention, Merocel packs were placed for 3 days to avoid the formation of synechiae between the turbinate and septum.

Cryotherapy. A standard nitrogen protoxide cryogenic nasal probe was applied along the free edge and then on the medial face of the inferior turbinate at 2 overlapping levels for 2 minutes, at a temperature of $-80^{\circ} \mathrm{C}$. Merocel nasal packing was left in each side for 3 days.

Submucosal Resection. Via a 3- to 4-mm incision on the head of the inferior turbinate, the submucosal tissue was dissected from the medial surface and inferior edge of the bone with an elevator. The excess cavernous tissue was resected with a Hartmann forceps, and the posterior end of the turbinate was resected. Merocel packing was used.

Submucosal Resection With Lateral Displacement. The above-mentioned submucosal resection technique was used, plus out-fracture and lateral displacement of the turbinal bone with a Goldman displacer.

TABLE 3. NASAL RESISTANCE DURING FOLLOW-UP ACCORDING TO ANTERIOR ACTIVE RHINOMANOMETRY

\begin{tabular}{lcccccc}
\hline \hline Treatment & 1st Year & 2nd Year & 3rd Year & 4th Year & 5th Year & 6th Year \\
\hline Turbinectomy & $0.30 \pm 0.09$ & $0.30 \pm 0.09$ & $0.45 \pm 0.10$ & $0.45 \pm 0.09$ & $0.40 \pm 0.11$ & $0.40 \pm 0.10$ \\
Laser cautery & $0.50 \pm 0.09$ & $0.55 \pm 0.10$ & $0.50 \pm 0.10$ & $0.60 \pm 0.14$ & $0.50 \pm 0.16$ & $0.55 \pm 0.14$ \\
Electrocautery & $0.80 \pm 0.13$ & $0.85 \pm 0.14$ & $1.20 \pm 0.33$ & $1.50 \pm 0.41$ & $1.20 \pm 0.46$ & $1.05 \pm 0.41$ \\
Cryotherapy & $0.60 \pm 0.11$ & $0.60 \pm 0.12$ & $0.90 \pm 0.26$ & $1.00 \pm 0.29$ & $0.90 \pm 0.31$ & $0.90 \pm 0.38$ \\
Submucosal resection & $0.45 \pm 0.09$ & $0.45 \pm 0.09$ & $0.50 \pm 0.11$ & $0.50 \pm 0.12$ & $0.45 \pm 0.15$ & $0.45 \pm 0.18$ \\
Submucosal resection with lateral & $0.45 \pm 0.09$ & $0.45 \pm 0.09$ & $0.45 \pm 0.10$ & $0.45 \pm 0.12$ & $0.45 \pm 0.14$ & $0.45 \pm 0.14$
\end{tabular}
displacement 
TABLE 4. NASAL VOLUME MODIFICATIONS DURING FOLLOW-UP ACCORDING TO ACOUSTIC RHINOMETRY

\begin{tabular}{|c|c|c|c|c|c|c|}
\hline Treatment & 1st Year & 2nd Year & 3rd Year & 4th Year & 5th Year & 6th Year \\
\hline Turbinectomy & $12.5 \pm 1.95$ & $11.8 \pm 2.15$ & $11.7 \pm 2.03$ & $11.8 \pm 2.01$ & $11.7 \pm 1.98$ & $11.8 \pm 2.11$ \\
\hline Laser cautery & $10.4 \pm 2.52$ & $9.5 \pm 2.37$ & $8.1 \pm 2.12$ & $7.4 \pm 1.93$ & $7.6 \pm 2.11$ & $7.5 \pm 1.92$ \\
\hline Electrocautery & $8.5 \pm 1.35$ & $7.3 \pm 1.28$ & $5.5 \pm 1.12$ & $4.5 \pm 1.13$ & $5.0 \pm 1.20$ & $4.8 \pm 1.31$ \\
\hline Cryotherapy & $9.3 \pm 1.31$ & $7.6 \pm 1.14$ & $5.7 \pm 1.03$ & $4.9 \pm 0.98$ & $5.0 \pm 1.05$ & $4.9 \pm 1.12$ \\
\hline Submucosal resection & $12.4 \pm 2.96$ & $10.2 \pm 2.05$ & $11.3 \pm 2.14$ & $11.4 \pm 2.41$ & $11.3 \pm 2.24$ & $11.4 \pm 2.35$ \\
\hline Submucosal resection with lateral & $11.5 \pm 2.34$ & $10.2 \pm 2.15$ & $11.1 \pm 2.47$ & $11.7 \pm 2.39$ & $11.5 \pm 2.36$ & $11.6 \pm 2.25$ \\
\hline
\end{tabular}
displacement

Data are mean values \pm SD in cubic centimeters. Mean volume before surgery was $4.8 \mathrm{~cm}^{3}$.

The patients were randomly assigned to 1 of the 6 therapeutic groups (Table 1); the 6 groups did not differ in regard to the severity of the disease before surgery.

The surgeons involved in the study performed a balanced percentage of each type of operation, using standardized techniques. Residents were not involved. In all cases, nasal packing was used without a topical antibiotic; all patients received a third-generation cephalosporin for 6 days. Informed written consent was signed by all of the patients. The study was conducted in accordance with the ethics committee of the University of Siena.

At yearly follow-up intervals, the above-mentioned set of examinations was repeated. All data were analyzed by Student's $t$-test.

\section{RESULTS}

Of the 382 patients initially enrolled in the trial, 348 were followed up, for a maximum of 6 years. Specifically, the follow-up was 6 years in 92 patients, 3 years in 82 patients, 2 years in 98 patients, and 1 year in 76 patients.

In 182 patients (40\%), allergic sensitization was found. Nasal hyperactivity was triggered by Dermatophagoides in 54 cases, by grass pollen in 18, and by both allergens in the remaining cases.

The complications that occurred in the 6 groups during the follow-up are listed in Table 2. Specifically, patients who underwent turbinectomy, laser cautery, electrocautery, and cryotherapy experienced chronic crusting in 34, 40, 39, and 40 cases, respectively; postoperative synechiae developed more frequently in the electrocautery group (21 cases). Bleeding was noted only in the submucosal resection group (with or without lateral displacement) and the turbinectomy group, reaching clinical significance in the latter group only.

Data regarding nasal resistance values measured by anterior active rhinomanometry and nasal volume values measured by acoustic rhinometry are summarized in Tables 3 and 4. We noted an initial postoperative improvement of the above-mentioned parameters in all groups $(\mathrm{p}<.001)$, whereas the duration of improvement significantly differed among groups. Specifically, in the groups treated with turbinectomy or submucosal resection (with or without lateral displacement), normal nasal patency was restored and remained during the follow-up.

In contrast, the patients who underwent electrocautery or cryotherapy experienced a progressive, statistically significant worsening of nasal resistance values $(\mathrm{p}<.005)$ and nasal volume values $(\mathrm{p}<.001)$; moreover, in the patients who underwent laser cautery, although nasal resistances improved and remained normal, nasal volumes were dramatically reduced during the 6 years $(\mathrm{p}<.001)$.

The results of mucociliary transport time and secretory $\operatorname{Ig}$ A concentration analyses are listed in Tables 5 and 6 . Only the patients with submucosal resection, with or without lateral displacement, achieved normalized parameters $(\mathrm{p}<.001)$. The submucosal resection group with lateral displacement achieved the best results.

TABLE 5. MUCOCILIARY TRANSPORT TIMES

\begin{tabular}{lcccccc}
\hline \hline Treatment & 1st Year & 2nd Year & 3rd Year & 4th Year & 5th Year & 6th Year \\
\hline Turbinectomy & $29 \pm 0.92$ & $28 \pm 0.73$ & $29 \pm 0.85$ & $29 \pm 0.95$ & $28 \pm 0.98$ & $29 \pm 0.82$ \\
Laser cautery & $27 \pm 1.78$ & $27 \pm 1.67$ & $26 \pm 1.83$ & $27 \pm 1.90$ & $27 \pm 1.84$ & $27 \pm 1.68$ \\
Electrocautery & $26 \pm 1.64$ & $25 \pm 1.57$ & $26 \pm 1.49$ & $25 \pm 1.93$ & $26 \pm 1.70$ & $26 \pm 1.53$ \\
Cryotherapy & $25 \pm 1.92$ & $26 \pm 2.15$ & $26 \pm 2.05$ & $25 \pm 1.93$ & $25 \pm 2.05$ & $26 \pm 1.89$ \\
Submucosal resection & $20 \pm 2.45$ & $20 \pm 2.36$ & $18 \pm 2.15$ & $20 \pm 2.41$ & $19 \pm 2.25$ & $18 \pm 2.34$ \\
Submucosal resection with lateral & $21 \pm 2.15$ & $18 \pm 1.83$ & $15 \pm 1.75$ & $15 \pm 1.68$ & $16 \pm 1.73$ & $15 \pm 1.67$
\end{tabular}

Data are mean values \pm SD in minutes. Normal mucociliary transport time is $13 \pm 2$ minutes. 
TABLE 6. SECRETORY IGA CONCENTRATIONS

\begin{tabular}{lrccccc}
\hline \hline Treatment & 1st Year & 2nd Year & 3rd Year & 4th Year & 5th Year & 6th Year \\
\hline Turbinectomy & $20 \pm 5.30$ & $15 \pm 4.60$ & $18 \pm 4.80$ & $20 \pm 5.50$ & $20 \pm 4.50$ & $21 \pm 5.80$ \\
Laser cautery & $10 \pm 2.40$ & $11 \pm 2.20$ & $15 \pm 2.90$ & $10 \pm 2.50$ & $12 \pm 2.65$ & $13 \pm 2.48$ \\
Electrocautery & $10 \pm 2.50$ & $10 \pm 2.60$ & $12 \pm 2.90$ & $18 \pm 3.10$ & $21 \pm 2.90$ & $23 \pm 2.54$ \\
Cryotherapy & $8 \pm 2.20$ & $12 \pm 2.80$ & $10 \pm 2.70$ & $15 \pm 2.80$ & $18 \pm 2.15$ & $20 \pm 2.20$ \\
Submucosal resection & $40 \pm 8.30$ & $35 \pm 7.80$ & $40 \pm 8.40$ & $53 \pm 9.20$ & $59 \pm 9.55$ & $57 \pm 8.40$ \\
Submucosal resection with lateral & $38 \pm 8.10$ & $40 \pm 8.60$ & $52 \pm 9.50$ & $70 \pm 12.80$ & $73 \pm 11.50$ & $75 \pm 12.70$
\end{tabular}

Data are mean values \pm SD in milligrams per $100 \mathrm{~mL}$. Normal secretory $\operatorname{IgA}$ concentration is 80 to $100 \mathrm{mg} / 100 \mathrm{~mL}$.

Table 7 summarizes the yearly patient judgments on their symptoms. The patients' subjective discomfort clearly followed the measured objective nasal parameters: symptoms worsened in the groups treated with electrocautery or cryotherapy, simultaneously with the worsening of nasal resistance and volume values. In contrast, in the patients treated with turbinectomy or submucosal resection, the symptom scores remained stable during the follow-up. Interestingly, the seriousness of symptoms (as stated by symptom scores) dramatically differed between the two groups $(\mathrm{p}<.001)$; the submucosal resection patients experienced a better quality of life. The addition of lateral displacement to the submucosal resection gave the best results (Fig 1).

\section{DISCUSSION}

Surgical treatment for inferior turbinate hypertrophy is based on the assumption that an increase in nasal airway volumes will lead to a better functional nasal airflow and improvement of the patients' symptoms. This is not completely true, because an overaggressive approach, although able to completely solve the ventilatory problem, interferes in a significant way with nasal physiology. In our study, turbinectomy gave excellent relief of nasal obstruction, but it was associated, as compared with the other techniques, with a higher percentage of crusting and bleeding (Table 2) and with more intense postoperative pain. Some authors ${ }^{18}$ have noted that a huge postoperative increase in nasal airflow corresponds to a decrease in the humidifying activity of the nasal mucosa with an excessive drying of nasal secretions and consequent crust formation. Moreover, our results in- dicate that the dramatic improvement in nasal airflow following complete resection of the inferior turbinates is accompanied by a decreased efficiency of mucociliary transport (Fig 2) and by a reduction in secretory $\operatorname{IgA}$ production (Table 6).

On the other hand, we demonstrated that laser cautery, electrocautery, and cryotherapy provided only short-term benefits in nasal resistance and volume (Tables 3 and 4). Moreover, these techniques produce mucosal damage that strongly interferes with nasal physiology (Tables 5 and 6). The few shortterm advantages variably associated with these three procedures (reduced bleeding, lack of postoperative pain, faster healing, etc) do not completely justify their use, when compared with the failure rate in achieving long-term outcomes.

According to our data, it clearly emerges that submucosal resection of the vascular tissue within the turbinate obtained an optimal long-term restoration of nasal patency (Tables 3 and 4), producing few postoperative complications (Table 2) and restoring mucociliary clearance and local secretory IgA production to a physiological level (Fig 2 and Table 6) that lasted for the full 6 years of follow-up.

The addition of lateral displacement of the inferior turbinate improved the long-term results. Although the difference between the group treated with submucosal resection only and the group treated with submucosal resection plus lateral displacement did not reach statistical significance, this supplementary surgical step improved the outcome without any additional risk.

TABLE 7. SYMPTOM SCORES

\begin{tabular}{lcccccc}
\hline \hline Treatment & 1st Year & 2nd Year & 3rd Year & 4th Year & 5th Year & 6th Year \\
\hline Turbinectomy & $21 \pm 2.43$ & $20 \pm 2.35$ & $22 \pm 2.72$ & $20 \pm 2.11$ & $21 \pm 2.14$ & $20 \pm 2.36$ \\
Laser cautery & $21 \pm 2.45$ & $24 \pm 3.10$ & $18 \pm 2.11$ & $20 \pm 2.23$ & $21 \pm 2.16$ & $20 \pm 2.16$ \\
Electrocautery & $20 \pm 2.25$ & $22 \pm 2.78$ & $26 \pm 3.76$ & $26 \pm 3.82$ & $27 \pm 2.91$ & $26 \pm 3.57$ \\
Cryotherapy & $20 \pm 3.11$ & $22 \pm 3.34$ & $27 \pm 3.85$ & $25 \pm 3.78$ & $26 \pm 3.22$ & $26 \pm 3.54$ \\
Submucosal resection & $10 \pm 1.59$ & $12 \pm 1.79$ & $12 \pm 1.81$ & $12 \pm 1.89$ & $13 \pm 1.88$ & $12 \pm 1.79$ \\
Submucosal resection with lateral & $10 \pm 1.43$ & $10 \pm 1.67$ & $10 \pm 1.85$ & $10 \pm 1.72$ & $11 \pm 1.62$ & $10 \pm 1.68$
\end{tabular}

Data are points of scale ranging between 5 and 30 (mean value \pm SD). 


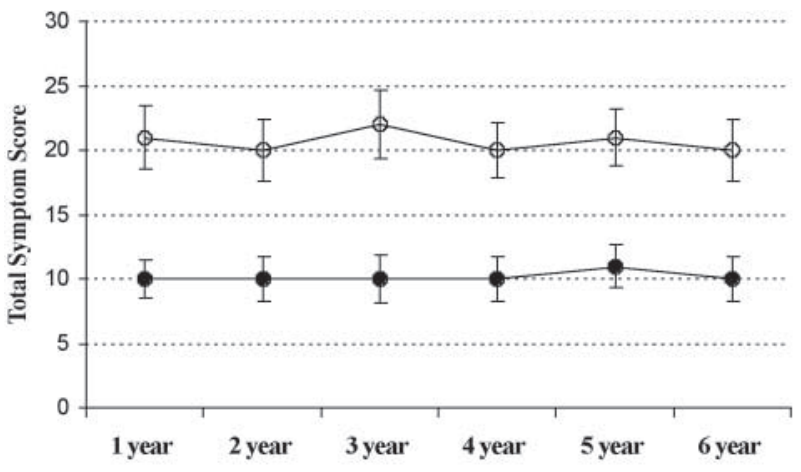

Fig 1. Symptom score modifications (yearly symptom scores; mean value \pm SD) during follow-up of turbinectomy (empty circles) versus submucosal resection with lateral displacement (filled circles). Symptom scores remained stable during follow-up in both groups. Seriousness of symptoms dramatically differed between two groups $(p<.001)$ during entire follow-up period.

Finally, we deliberately neglected to explore in the present study two recent and interesting minimally invasive surgical approaches to inferior turbinate hypertrophy, namely, radiofrequency ${ }^{19}$ and coblation turbinate reduction. ${ }^{20}$

In our department, we currently treat patients with radiofrequency and coblation turbinate reduction with good results, but our experience with these procedures includes no more than 2 years of follow-up. For this reason, we decided not to consider as definitive the good results we obtained with these two techniques and did not include these data in the present study.

In our experience and according to the literature, $, 4,8$ rhinosinusal physiological mechanisms, after a surgical approach to this region, need at least 2 years to be restored. Specifically, nasal patency may begin to worsen, after surgical reduction of inferior turbinate hypertrophy, starting from the third or fourth year of follow-up. For this reason, patients surgically treated for this kind of disorder (whatever the chosen technique) have to be followed up for at least 3 years to show reliable results.

\section{CONCLUSIONS}

The ideal surgical approach to the inferior turbinate should be limited to the erectile submucosal tissue and to the bony turbinate: the reduction of bone

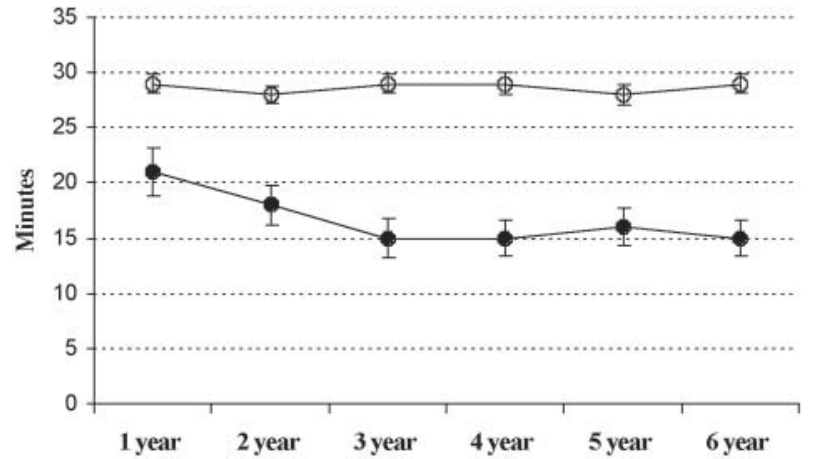

Fig 2. Mucociliary transport time modifications (yearly mucociliary transport times; mean value \pm SD) during follow-up of turbinectomy (empty circles) versus submucosal resection with lateral displacement (filled circles). Mucociliary transport times of turbinectomy group remained altered during entire follow-up period, whereas in submucosal resection with lateral displacement group, this parameter progressively turned back to physiological range $(\mathrm{p}<.001)$.

size will create more respiratory space, the surgical maneuvers performed on submucosal tissues will create scars able to minimize the submucosal engorgement in patients with allergic rhinitis, and the preservation of the mucosa will minimize interference with the physiological activities of this region.

Considering the actual international state of the art of turbinate surgery, and according to data provided by the present 6-year follow-up study, we believe that the technique nearest to the above-mentioned ideal one is submucosal resection with lateral displacement. Undoubtedly, the elevation of a mucoperiosteal flap requires a higher level of technical ability than a mere full-thickness excision; for this reason, many surgeons prefer turbinectomy to submucosal resection, sacrificing, in this way, the possibility of correctly restoring the nasal physiology.

In conclusion, considering that as discussed above, the quality of life of patients affected by chronic nasal obstruction seems to be related more to the restoration of nasal physiology than to the simple increase of nasal volume, we recommend, in spite of the greater surgical skill required, submucosal resection combined with lateral displacement as the first-choice technique for the treatment of nasal obstruction due to hypertrophy of the inferior turbinates.

ACKNOWLEDGMENT - The authors thank Dr George A. Gates (Department of Otolaryngology-Head and Neck Surgery, University of Washington Medical Center) for excellent editorial support.

\section{REFERENCES}

1. Bridger GP, Proctor DF. Maximum nasal inspiratory flow and nasal resistance. Ann Otol Rhinol Laryngol 1970;79:4818 .

2. Juniper EF. Impact of upper respiratory allergic diseases on quality of life. J Allergy Clin Immunol 1998;101(suppl): S386-S391.
3. Passàli D, Lauriello M, Mezzedimi C, Passàli GC, Bellussi L. Natural history of allergic rhinitis: a review. Clin Appl Immunol Rev 2001;1:207-16.

4. Hol MK, Huizing EH. Treatment of inferior turbinate pathology: a review and critical evaluation of the different techniques. Rhinology 2000;38:157-66. 
5. Ophir D, Schindel D, Halperin D, Marhak G. Long-term follow-up of the effectiveness and safety of inferior turbinectomy. Plast Reconstr Surg 1992;90:980-7.

6. Elwany S, Harrison R. Inferior turbinectomy: comparison of four techniques. J Laryngol Otol 1990;104:206-9.

7. Bergler WF, Sadick H, Hammerschmitt N, Oulmi J, Hormann K. Long-term results of inferior turbinate reduction with argon plasma coagulation. Laryngoscope 2001;111:1593-8.

8. Lagerholm S, Harsten G, Emgard P, Olsson B. Laserturbinectomy: long-term results. J Laryngol Otol 1999;113:52931.

9. Talaat M, el-Sabawy E, Baky FA, Raheem AA. Submucous diathermy of the inferior turbinates in chronic hypertrophic rhinitis. J Laryngol Otol 1987;101:452-60.

10. Moore JR, Bicknell PG. A comparison of cryosurgery and submucous diathermy in vasomotor rhinitis. J Laryngol Otol 1980;94:1411-3.

11. Principato JJ. Chronic vasomotor rhinitis: cryogenic and other surgical modes of treatment. Laryngoscope 1979;89: 619-38.

12. Motta G, Pucci V, Villari G, Motta G Jr, Salzano FA. Cryosurgery in the treatment of hypertrophic chronic rhinitis. In: Passàli D, ed. Around the nose. Firenze, Italy: Conti Tipocolor, 1988:189-203.
13. House HP. Submucous resection of the inferior turbinate. Laryngoscope 1951;61:637-48.

14. Friedman M, Tanyeri H, Lim J, Landsberg R, Caldarelli D. A safe, alternative technique for inferior turbinate reduction. Laryngoscope 1999;109:1834-7.

15. O’Flynn PE, Milford CA, Mackay IS. Multiple submucosal out-fractures of inferior turbinates. J Laryngol Otol 1990; 104:239-40.

16. Galetti G, Dallari S, Galetti R. Turbinoplasty: personal technique and long-term results. ORL J Otorhinolaryngol Relat Spec 1991;53:111-5.

17. Passali D, Bellussi L, Bianchini Ciampoli M, De Seta E. Experiences in the determination of nasal mucociliary transport time. Acta Otolaryngol (Stockh) 1984;97:319-23.

18. Salam MA, Wengraf C. Concho-antropexy or total inferior turbinectomy for hypertrophy of the inferior turbinates? A prospective randomized study. J Laryngol Otol 1993;107:11258.

19. Coste A, Yona L, Blumen M, et al. Radiofrequency is a safe and effective treatment of turbinate hypertrophy. Laryngoscope 2001;111:894-9.

20. Bortnick DP. Coblation: an emerging technology and new technique for soft-tissue surgery. Plast Reconstr Surg 2001;107: 614-5. 